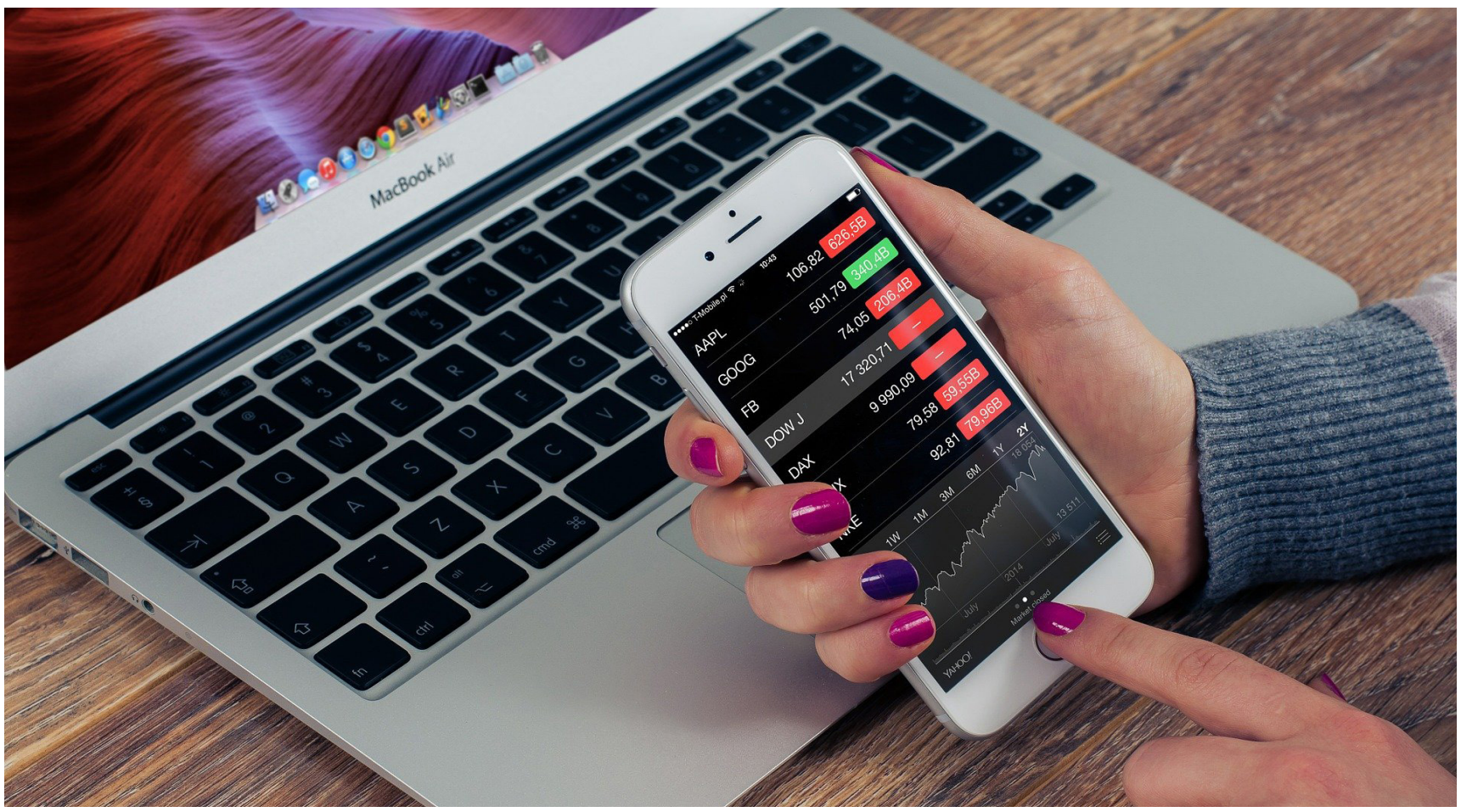

\title{
Virtualización en procesos de control de pedidos de tuberías con Vmware.
}

\section{Virtualization in pipe order control processes with Vmware.}

Javier Lima-Morales

Ingeniero Electrónico, jalimo2k12@hotmail.com, Fundación Tecnológica

Antonio de Arévalo, Cartagena de Indias, Colombia

Luis Alejandro Rojas

Especialista en Gestión de Sistemas Eléctricos de Potencia.

luis.rojas@tecnar.edu.co, Fundación Tecnológica Antonio de Arévalo,

Cartagena de Indias, Colombia

Marcos Carreazo-Pérez

Ingeniero Electrónico, mcarriezo@hotmail.com, Fundación Tecnológica

Antonio de Arévalo, Cartagena de Indias, Colombia 
Cómo citar: J. Lima-Morales, L.A. Rojas y M. Carreazo-Pérez, "Virtualizalización en procesos de control de pedidos de tuberías con VMware”, Sostenibilidad, Tecnología y Humanismo, vol. 11, no. 1, 45-53, 2018.

\section{Resumen}

En el presente trabajo se explicará la virtualización en procesos de control de pedidos de tuberías con VMWare, a través, de la implementación de multiplex, servidores y desktop virtuales, que ayudan a controlar los procesos que se necesitan para el pedido de la tubería en la planta, radicando esto, en un gran avance en términos informáticos. El objeto principal de este artículo es dar a conocer la utilidad de la virtualización en empresas con producciones en masas. Esta gran herramienta tecnológica posibilita alojar virtualmente varias computadoras en una maquina real, sacando el mayor provecho del software y ahorrando en hardware. Innovar es la clave de esta herramienta, permite mejorar y agilizar los procesos dedicados a la manufactura de tubería de alta presión; de igual manera, se mostrara su debida aplicabilidad, permitiendo renovar el modelo actual de trabajo; creando una arquitectura extremadamente flexible y amigable en los procesos internos de la producción; se podrá observar la infraestructura requerida y, su debida implementación. La virtualización como estrategia de negocios es nuevamente usada, ya que la misma da como resultado una producción eficaz, ahorro energético, reducción de componentes electrónicos, recuperación rápida de datos, y la eficiencia en los procesos cotidianos de las empresas.

Palabras clave: Virtualización, infraestructura, control, VMWare, multiplex.

\section{Abstract}

This work will explain the virtualization of pipeline order control processes with VMWare, through the implementation of virtual multiplex, servers and desktop, which help to control the processes needed for the ordering of the pipeline in the pipeline. plant, rooting this, in a breakthrough in computer terms. The main purpose of this article is to publicize the usefulness of virtualization in companies with mass productions. This great technological tool makes it possible to host virtually several computers in a real machine, making the most of the software and saving on hardware. Innovating is the key to this tool, it allows improving and streamlining the processes dedicated to the manufacture of high pressure pipes; in the same way, its due applicability will be shown in real time, allowing to renew the current work model; By creating an extremely flexible and friendly architecture in the internal production processes, the required infrastructure and its proper implementation can be observed. Virtualization as a business strategy is used again as it results in efficient production, energy savings, reduction of electronic components, rapid data recovery, and efficiency in the daily processes of companies.

Key words: Virtualization, infrastructure, control, VMWare, multiplex 
Introducción

Hoy en día el entorno empresarial se mueve a ritmo vertiginoso, consecuencia de la globalización tecnológica que cada día se afianza más en el mercado, así lo refleja [1] un mundo altamente complejo como el actual expresa una profunda crisis; dando espacio a la búsqueda de nuevas estrategias tecnologías y comunicación; presentando opciones para el mejoramiento de los procesos repetitivos y cotidianos, innovando con nuevas herramientas tecnológicas, en busca siempre de la fluidez de la información, eficacia en los procesos y eficiencias en los recurso usados.

De modo que, el trabajo por resultado hace obligatorio que los tiempos de respuestas cada vez sean más cortos, en tanto, [1] expresa la necesidad de detectar y determinar cuáles serían los elementos intangibles que permitirían incrementar la competitividad de las empresas, por ellos se afianza la búsqueda constante de herramientas a nivel tecnológicas para optimizar los procesos y ahorrar recursos, se hace más presente la idea de retomar una estrategia tecnológica como lo es la virtualización, definido por [2] como la idea de mejorar la utilización de recursos tecnológicos mediante una agrupación común de éstos y que se pueden llegar compartir con cualquier persona en el mundo. Aun cuando esta tecnología no es nada novedosa, es de suma utilidad por las bondades que permite en los procesos industriales.

Así mismo, [3] afirman que "con la evolución tecnológica constante, es natural que las instituciones se enfrenten a cambios drásticos de paradigmas con respecto a la forma en que funcionan, se administran y se entregan los servicios en las infraestructuras tecnológicas", por lo tanto se realiza este artículo para mostrar una panorámica de la virtualización en los procesos de control de pedidos de tuberías con VMWARE, características, su aplicación y la estructura necesaria para su implementación.

Virtualización.

Atores como [4] expresan que la virtualización es una estrategia que, básicamente permite integrar y compartir recursos, Coadyuvando a los procesos internos de cualquier empresa que está inmersa en el uso de la tecnología, fungiendo como brazo ejecutor de los procesos rutinarios de la empresa.

La virtualización es sinónimo de aprovechamiento de recursos informáticos, ya que nos permite emular en una sola maquina múltiples sistemas operativos con su propio hardware virtual como su memoria Ram, CPU, discos duros con tecnología Hypervisor, es decir, es un sistema operativo anfitrión y poder alojar huéspedes sin afectarse entre ellos, tanto así que no detectan que están compartiendo la misma casa. "El espacio virtual simboliza el paradigma que tiende a ser dominante en el mundo actual, tendencia que se materializa en la creciente emergencia de mecanismos y procedimientos que virtualizan las dinámicas sociales" [5].

Con base a esta premisa, [6] refleja que la idea principal de la virtualización es que se pueda instalar un sistema operativo "virtual" sobre otro sistema base. Esto permite crear tantas máquinas virtuales como sea necesario para cubrir las necesidades de la industria.

Una de las ventajas de la virtualización es el impacto directamente en la rentabilidad y productividad de la empresa, dejando atrás tras los procesos manuales de la manufactura dándole más importancia a los tiempo de producción; con base a ello, [7] precisa que la administración tradicional, se trata principal y estrictamente de controlar, registrar, ordenar y mantener; sin mostrar la mínima necesidad 


\section{8}

de algún cambio en los procesos manuales que pudieran dar respuesta a corto plazo y bajo costo; tal vez la gran inversión a nivel monetario, no se muestra la intención de un cambio innovador, puesto que usar multiplex equipos de cómputo para realizar este control era muy costoso, adicional a ello se derivaba unos temas de infraestructura.

VM maquina virtuales.

Actualmente somos testigos de un cambio de paradigma. La vida cotidiana se va impregnando inextricablemente del flujo informativo que surge del espacio virtual [5]. Una de las tecnología de información más potentes hoy en día es la virtualización, y para requiere de unas Máquinas Virtuales, definidos como sistemas operativos que trabajan en paralelo por decirlo así, al sistema operativo del host el cual proporciona los recursos de hardware y configuración, permitiendo emular multiplex aplicaciones en un solo ordenador compartiendo una sola estación de trabajo; así lo refleja [6] "consiste instalar sistemas operativos de forma virtual con base a otro llamado "anfitrión" o Host ". La tendencia que a finales del siglo XX apuntaba a una digitalización paulatina de la cultura no ha hecho sino incrementarse drásticamente en la última década.

Así mismo, [8] afirman que una máquina virtual es un programa informático que crea un entorno virtual entre el sistema operativo $y$ el hardware para que el usuario final pueda ejecutar aplicaciones en una máquina abstracta; realizando varias tareas a un mismo momento, sin pérdida de tiempo ni información; aportando rapidez y eficiencia en los procesos productivos en las empresas según sean los casos de producción en masas (figura 1). "Actualmente somos testigos de un cambio de paradigma". La vida cotidiana se va impregnando inextricablemente del flujo informativo que surge del espacio virtual, y la tendencia que a finales del siglo XX apuntaba a una digitalización paulatina de la cultura no ha hecho sino incrementarse drásticamente en la última década" [9].

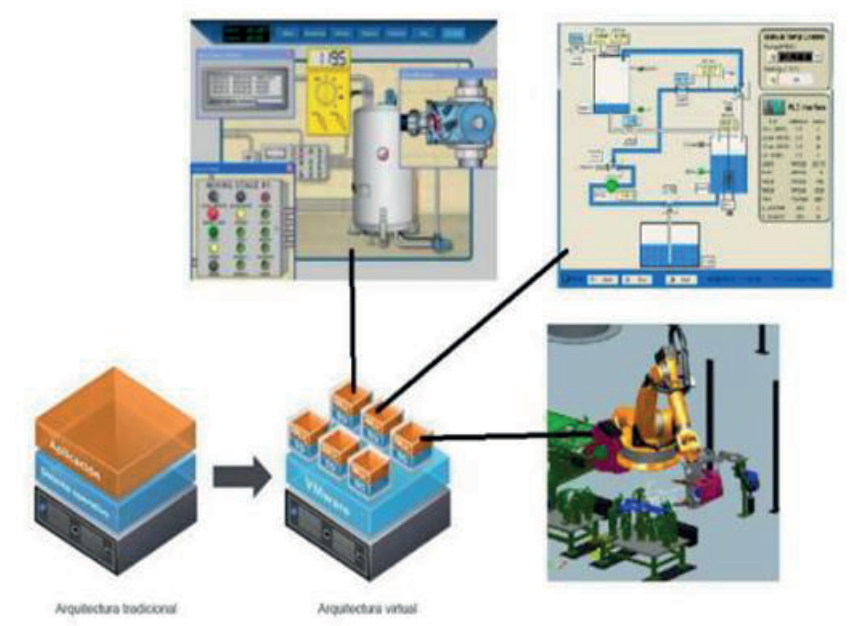

Figura 1. Estructura virtual

VMware.

Autores como [10] sostiene que El uso de la tecnología de la virtualización no es una simple especulación del futuro de las computadoras; siendo esto evidente con la rentabilidad que obtienen las diferentes compañías más potentes este mercado, siendo una de ellas VMWare, esta cuya empresa ha sido pionera en la creación de un servidor que permita corre distintos sistemas operativos como máquinas virtuales en un solo ordenador; indiscutiblemente es una de las herramientas de virtualización más potente que puedes utilizar. "Hoy en día, el uso más claro para la virtualización y emulación se centra en servidores" [11].

Con base a esta premisa, [12] sostienen que VMware es unas de las herramientas más innovadoras del mercado que permite diseñar una nube privada ajustadas a las necesidades de cada negocio, con el podrás virtualizar fácilmente cualquier sistema operativo Windows o Linux, sin necesidad de particionar 
tus discos duros, ni reiniciar el sistema. Además cuenta con una opción que te permite virtualizar tu propio ordenador, para así poder hacer cuantas pruebas y experimentos consideres oportunos.

De igual manera [13] sostiene que "existe una versión de VMWare pensada para servidores que no necesita un sistema operativo anfitrión", convirtiendo a este sistema operativo en uno de los más usados a nivel mundial para virtualizaciones de computadores personales a nivel mundial; esto ha hecho que la idea de virtualizar y emular madure cada día, y pueda usarse sin temor alguno (figura 2).
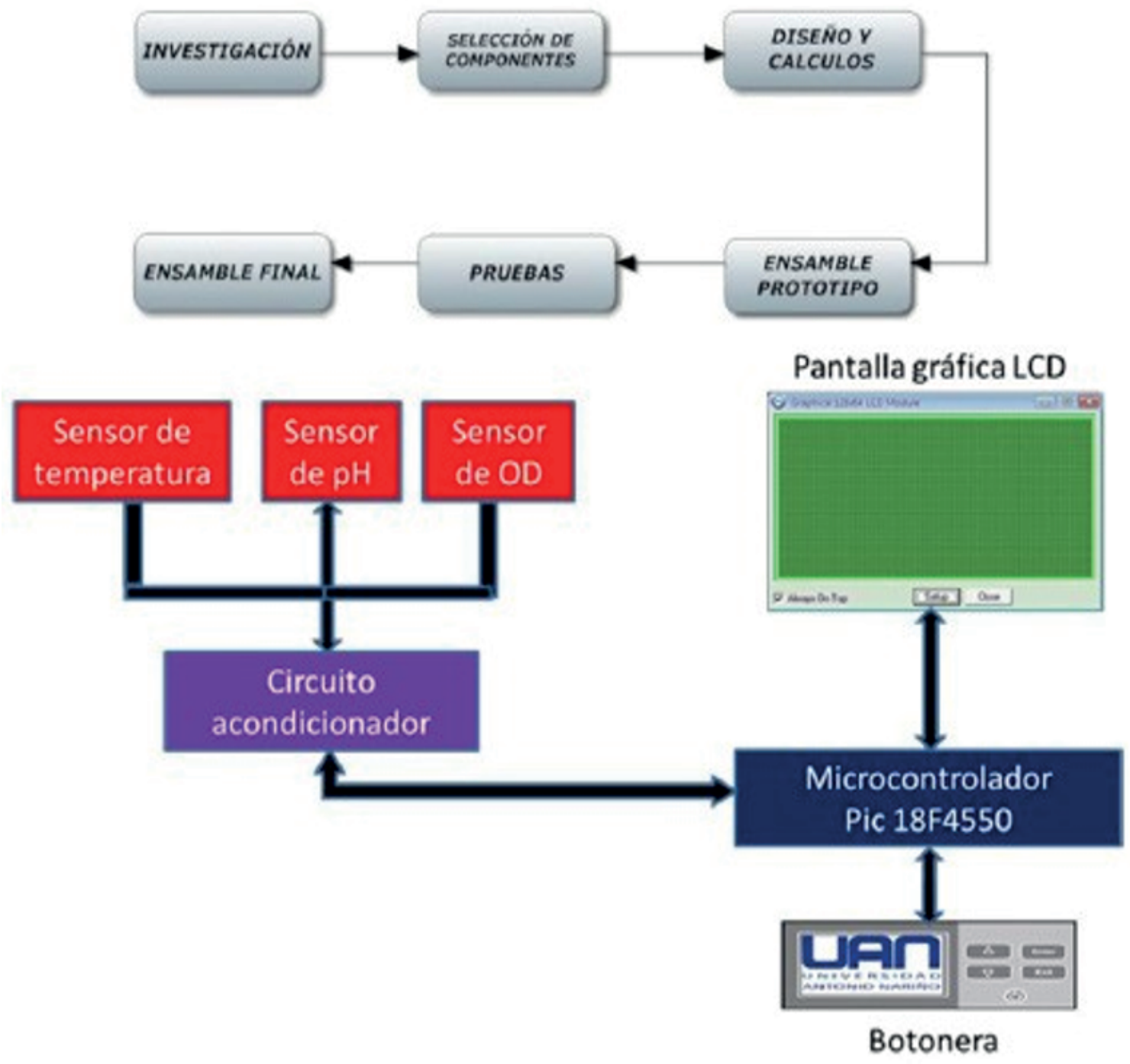

Figura 2. Software de Virtualización 


\section{0}

Aplicación.

En los procesos de manufactura de tubería desde hace unos años se ha implementado nuevas tecnologías de información con el fin de mejorar los procesos de control de pedido de tuberías, de igual manera, [4] afirman que las $\mathrm{TI}$ como la virtualización se han convertido en el elemento fundamental para crear infraestructuras dinámicas en ambientes empresariales; dando como consecuencia la automatización industrial que va de la mano con software desarrollado a medida que se implementa en servidores según el proceso y en desktop para que los operadores en turno en toda la áreas productiva en la planta. "La realidad virtual es asociada por la mayoría de las personas con "algún tipo de casco, guante o algún tipo de traje especial que lleva al usuario a vivir un mundo de fantasía" [13].

No obstante, la automatización de los procesos empresariales con producción en masas, muchas veces no optaba por innovar tecnológicamente, por temas del presupuestos adicionales que se necesita para toda esta infraestructura tradicional en hardware, cableado de red, acometidas eléctricas y personal capacitado para soportar todos estos temas de hardware y software, con la implementación de la virtualización se ha logrado reducir los costos en infraestructura para tal fin, así lo afirma [14] la virtualización como tendencia propicia la automatización de los procesos como medio de reducir costes operación y responder con mayor agilidad a las oportunidades de mercado.

Anteriormente, los procesos se regían por una planeación rígida, que en algunos casos debía ser modificada por temas de prioridades que no está programada, generada por clientes vip, porque se tenía que reprogramar los equipos en planta uno a la vez. El autor [6] asegura que una de las ventajas de la virtualización es que los usuarios ejecutan múltiples sistemas al mismo tiempo en sólo un servidor físico, pero con la independencia suficiente como para simular una máquina para cada uno de ellos, ahí se cargan las aplicaciones envían a los diferentes dispositivos en su propio lenguaje y estructura de programación a los plc, variadores de velocidad, arrancadores, cortadoras, tornos cnc, bandas transportadoras y dispositivos que controlan las temperaturas de los hornos, todo esto en tiempo real (figura 3).

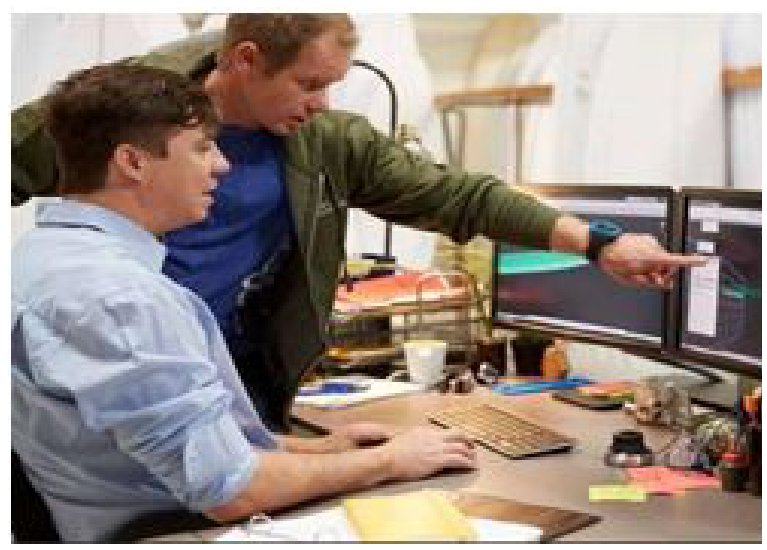

Figura 3. Software de virtualización de proceso.

Infraestructura.

Para la implementación de la infraestructura se realizó una etapa de diseño donde se tomaron en cuenta cuales eran los requerimiento actuales en temas de virtualización; procesamiento, memoria, almacenamiento, conectividad, estructura física, sistema de respaldo de energía a los cuales debían apuntar (figura 4). "La tendencia que a finales del siglo XX apuntaba a una digitalización paulatina de la cultura no ha hecho sino incrementarse drásticamente en la última década" [5]. 


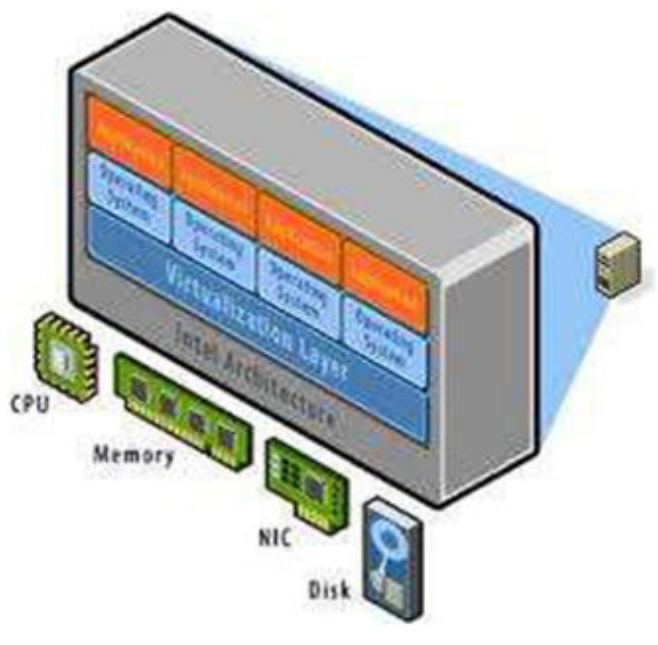

Figura 4. Infraestructura Virtual.

De igual manera, es importante destacar que ésta ingeniería de detalles que se llevó a cabo; evitó la compra de equipamiento que con el tiempo no iba a ser suficiente para suplir la necesidades operativas.

Inicialmente se definió la ubicación física en la planta de estos equipos, a lo cual se dio solución rápidamente porque los nuevos equipos entraban a hacer la renovación tecnológica de servidores que cumplieron con el tiempo de servicio y garantía estipulada por la empresa. Se reutilizo una ups de 10kVA, se implementó un cableado utp categoría $6^{\text {a }}$ enlazando el cableado hacia los switch cisco 2960x y el sistema de aire acondicionado de 48000 BTU de este nodo para servidores, esto garantizaba el tema de condiciones físicas y ambientales para su funcionamiento, agregándole seguridad y confiabilidad al sistema.

Si bien es cierto, la virtualización está basada en una estructura lógica que administra recursos físicos que están especialmente diseñados para la interacción entre estos dos elementes, por tal motivo, la elección de la plataforma de virtualización se realizó tomando en cuenta tres puntos: Estabilidad, Compatibilidad y Soporte 7x24 horas; el grupo asignado a la implementación de la tecnología, optó por la plataforma de virtualización VMWARE la cual cuenta con varias estructuras lógica, así comolo señala [10] el hipervisor o el contenedor como sistema operativo anfitrión van con los otros sistemas operativos invitados y es ahí donde se crea una capa virtual que le hace creer a los invitados que ellos tienen los recursos físicos con que cuenta la computadora.

Por consiguiente, se utiliza la línea de servidores HP proliant dl 360 generación 7 que tiene las siguientes características: Procesamiento con tecnología xeon hyper threading de $2.66 \mathrm{ghz} / 12 \mathrm{mb}$ cache 13 y soporta 2 procesadores, memoria de 192 GB ddr3, 5 conexiones a la red integradas a una velocidad de 1GB incluido ilo Almacenamiento 8 discos hot plug sff sata/sas 10TB hdd (figura 5). "En todo desarrollo, mantenimiento o implementación de un software los integrantes del equipo se comunican unos con otros, con los usuarios, con el cliente y otros especialistas; es por ello que la comunicación que se establece es compleja" [15].
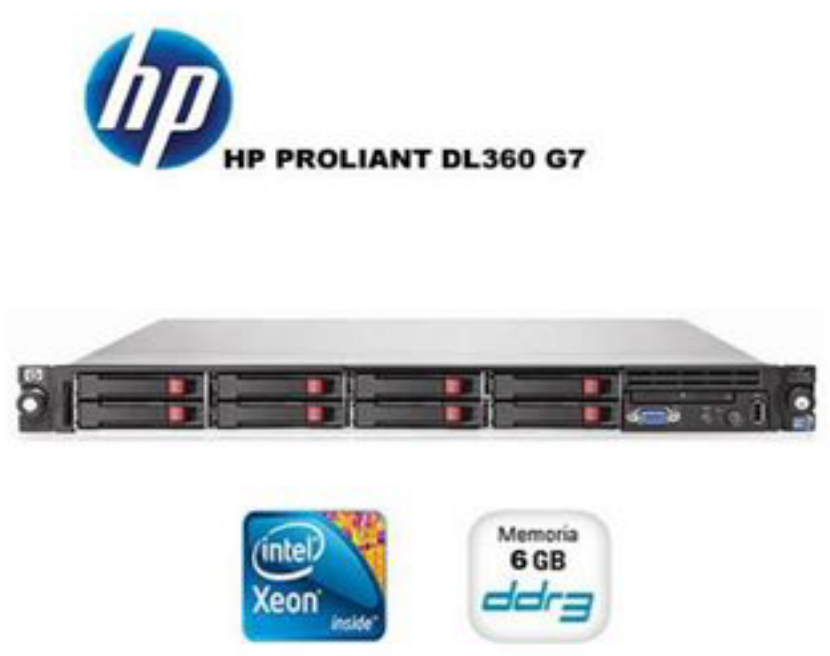

Figura 5. Infraestructura Virtual

Implementación.

Para la implementación se utilizaron 4 servidores del tipo proliant donde se instaló 


\section{2}

VMWare ESX versión 5.5 como sistema operativo convirtiendo en un host iESX el cual registra todos los recursos disponibles en ese servidor. Los pasos siguientes se centran en asignarle las ip's en el segmento de red preestablecían en la vlan asignada de modo estático, se realiza el conexionado a la red de datos y desde este punto es visible en la red. la plataforma de virtualización VMware ESXi, acto seguido, se realiza la configuración del switch virtual para conectar la máquinas virtuales a través de la red de datos interna que crea VMWare por defecto, luego de terminar con esta configuración, se crearon los primeros servidores y desktop virtuales para realizar las pruebas de software en la consola de administración remota del ESXi, al terminar las pruebas, se crearon 54 VMs entre VM tipo servidor y VM tipo desktop que se requerían según los estipulado en la investigación para el control de los procesos de control de pedido de tuberías, este trabajo fue tomado como referencia para nuevos desarrollos.

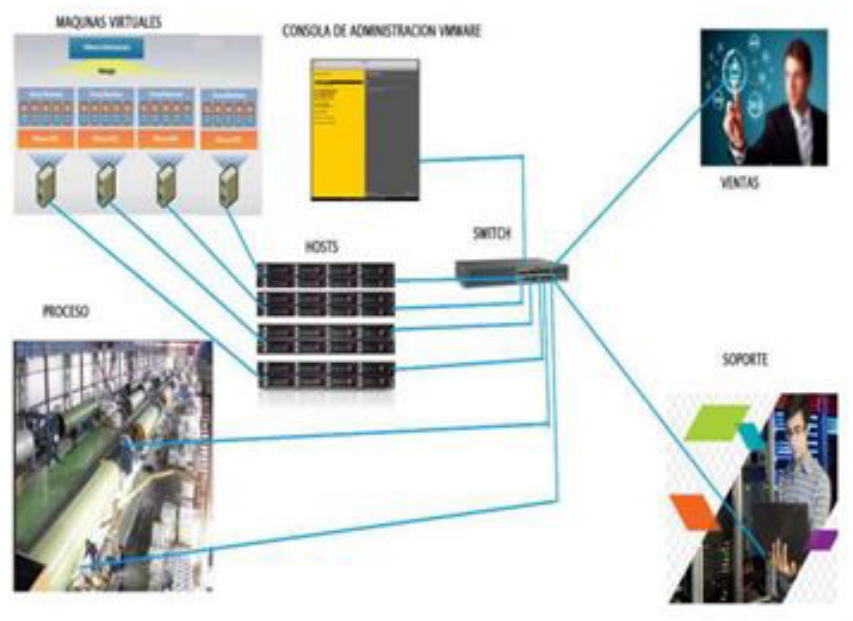

Figura 6. Infraestructura virtual del proceso de la planta.

- Mejoras en la interoperabilidad.

- Cambios rápidos al modificar los pedidos.

- Disminución de hardware Control de calidad

- Disminución del personal de soporte

- La capacidad de trabajar con múltiples software de los fabricantes en los dispositivos e instrumentos, implementado en la industria sin afectar otras aplicaciones.

- Mejor control en los procesos Control de alertas.

- Disminución en costos.

-Centralización de control en los procesos.

\section{Conclusiones}

Los beneficios que aporta la virtualización con VMWare del control de pedido de tuberías se traducen en el incremento de la eficacia, la eficiencia energética, la reducción de costes y una mayor capacidad de respuesta. Por lo tanto, es una opción que debe ser considerada por las empresas que deseen conseguir estas enormes ventajas y no se quieran quedar rezagadas con respecto a los avances tecnológicos.

Por lo tanto, resulta clave contar con la asesoría técnica especializada al momento de emprender una nueva investigación de virtualización, que es uno de los puntos que se tomó en cuenta para la implementación con VMWare.

De igual manera, en el mercado existen compañías expertas en la planificación y ejecución de "planes virtuales". Elegir la más apropiada permitirá conseguir los mejores resultados, el mejor aprovechamiento de los recursos y la mayor satisfacción del cliente final, definitivamente usar máquinas virtuales, dejando de ser una opción para convertirse en una necesidad para la solución el aprovechamiento de los recursos.

\section{Referencias}

[1] M.G. García Sandoval, H.D. Ariza Torrado, M.L. Pinzón y A.S. Flórez Fuentes, "BUENAS PRÁCTICAS APLICADAS A LA IMPLEMENTACION COLABORATIVO DE APLICATIVOS WEB". Mundo FESC, vol. 5, no. 10, pp. 27-30, 2016. Recuperado a partir de 
https://www.fesc.edu.co/Revistas/OJS/index. php/mundofesc/article/view/67

[2] M. Arias, "Percepción General De La Virtualización De Los Recursos Informáticos. InterSedes", Revista de las Sedes Regionales, vol. IX, no. 17, 2008

[3] J. Rojas, J. Forero, C. Montenegro y P. Gaona $\mathrm{P}$, "Hacia la virtualización de escritorios para la entrega de ambientes académicos basados en DaaS", Revista Logos, Ciencia \& Tecnología, vol. 7, núm. 2, pp. 85-96, 2016

[4] Y. Romero, K. García, "Virtualización InterSedes”, Revista Telem@tica, vol. 10, no. 3, 2011

[5] J. Fajardo J, "Virtualización de la producción académica en la Facultad de Arquitectura de la Universidad de Cuenca (Ecuador). ASRI - Arte y Sociedad", Revista de Investigación, no.7, 2014

[6] L. Ulloa, "La virtualización y su impacto en las ciencias computacionales", Revista Digital Lámpsakos, no. 2, pp. 118-121, 2009.

[7] E. Solorzano, La Administración Tradicional, 2011, [En línea]. Disponible en: http:// imgelismarsolorzano.blogspot.com.co/2011/04/ administracion-tradicional.html

[8] L. Montserrat, A. Ruiz, J. Pérez y J. Cano, Monografico; Máquinas virtuales, 2007, [En línea]. Disponible en: http://recursostic. educacion.es/observatorio/web/es/software/ software-general/462-monografico-maquinasvirtuales

[9] J. Guzmán-Luna, I. Torres I y J.F Alvrez, "Propuesta de un generador de aplicaciones educativas basadas en televisión digital usando arquitectura de cómputo en la nube", Revista Colombiana de Tecnologías de Avanzada, vol 2, no. 24, 2014
[10] L. Ordóñez L, “La tecnología de virtualización en las computadoras", CienciaUAT, vol. 3, núm. 4, pp. 56-59, 2009

[11] P. Gómez, "Máquinas Virtuales en las clases de Informática, Grupo de Aplicaciones de Inteligencia Artificial Facultad de Informática Universidad Complutense de Madrid”, 2008, [En línea]. Disponible en: http://bioinfo. uib.es/ joemiro/aenui/procJenui/Jen2006/ prDef0096_fad6f4e614.pdf

[12] E. Marchionni y O. Formoso, Virtualización con MVware. 1era edición. Buenos Aires: Fox Andina, 2012.

[13] B. Gros Salvat, "Metodología para el análisis de espacios virtuales colaborativos", RED Revista de Educación a Distancia, número 16, 2006

[14] C. Giraldo, "Virtualización como mejor práctica para minimizar las caídas de los sistemas de información en empresas de Retail en Colombia", 2009. [En línea]. Disponible en: http://hdl.handle.net/10654/13213.

[15] O. Molina, F. Fernández y J. Zambrano, "Reflexiones sobre la virtualización de la formación de habilidades investigativas en los estudiantes vinculados al desarrollo de software", Rev. Cubana Edu. Superior, vol. 36, no.3, 2017 